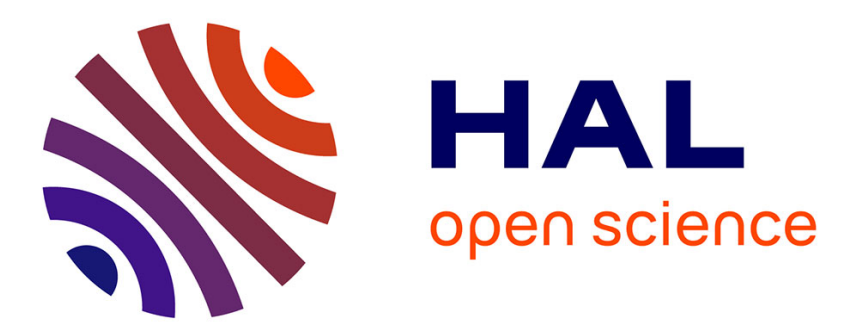

\title{
A constitutive equation for entangled linear polymers inspired by reptation theory and consistent with non-equilibrium thermodynamics
}

Adrien Leygue, Anthony Beris, Roland Keunings

\section{- To cite this version:}

Adrien Leygue, Anthony Beris, Roland Keunings. A constitutive equation for entangled linear polymers inspired by reptation theory and consistent with non-equilibrium thermodynamics. Journal of Non-Newtonian Fluid Mechanics, 2001, 101 (1-3), pp.95-111. 10.1016/S0377-0257(01)00143-4 . hal01004691

\section{HAL Id: hal-01004691 \\ https://hal.science/hal-01004691}

Submitted on 11 Apr 2018

HAL is a multi-disciplinary open access archive for the deposit and dissemination of scientific research documents, whether they are published or not. The documents may come from teaching and research institutions in France or abroad, or from public or private research centers.
L'archive ouverte pluridisciplinaire HAL, est destinée au dépôt et à la diffusion de documents scientifiques de niveau recherche, publiés ou non, émanant des établissements d'enseignement et de recherche français ou étrangers, des laboratoires publics ou privés. 


\title{
A constitutive equation for entangled linear polymers inspired by reptation theory and consistent with non-equilibrium thermodynamics
}

\author{
A. Leygue, A.N. Beris, R. Keunings \\ CESAME, Division of Applied Mechanics, Université catholique de Louvain, \\ Bâtiment Euler, B-1348 Louvain-la-Neuve, Belgium
}

\begin{abstract}
Using the single generator bracket formalism of non-equilibrium thermodynamics, we build a thermodynamically consistent constitutive equation of the differential type for linear entangled polymers. The starting point of our developments is the MGI rheological model proposed recently by Marrucci et al. [Rheol. Acta 40 (2) (2001) 98], which can be viewed as a modification of the classical Doi-Edwards reptation theory that includes convective constraint release and a modified strain measure. The proposed constitutive equation has an additional parameter which governs the dissipative part of the model. The MGI model is recovered as a particular case, but with a stress-conformation relationship which contains an additional term suggested by non-equilibrium thermodynamics. Predictions of the proposed model in steady and transient shear flows are shown to be in qualitative agreement with experimental observations.
\end{abstract}

Keywords: Non-equilibrium thermodynamics; Reptation theory; Linear polymers; Constitutive equation

\section{Introduction}

Since the introduction by De Gennes [2] of the reptation picture, a number of successful molecular theories have been able to describe with increasing accuracy the linear rheology of entangled linear polymers. The original idea is to consider the polymer chain confined in a tube formed by the topological constraints, or entanglements, between the chain and the surrounding polymer chains. The dominant relaxation mechanism comes from the reptation of the chain out of the confining tube. Considering chain length fluctuations, it is possible to predict with reasonable accuracy the linear viscoelastic properties of linear monodisperse polymers. The prediction of non-linear rheological properties is far less accurate, 
however. In the classical Doi-Edwards (DE) theory [3], the tube is deformed affinely by the flow and its segments are gradually destroyed and renewed as the chain slowly reptates out of the tube. It is also assumed that the chain-retraction mechanisms are so fast that the chain always has its equilibrium length. Among the successes of the classical DE theory, we find the prediction of the plateau modulus and of the damping function in response to a step strain. The DE model also predicts a non-zero second normal stress difference in shear flows. There are nevertheless major features that the DE model fails to predict, even qualitatively [4].

1. The transient response of DE in startup of shear flow has an overshoot in the shear stress but none for the first normal stress difference, while large overshoots both in shear and normal stresses are observed experimentally. Furthermore, DE predicts the overshoot in shear stress to occur at a strain which is independent of the shear rate, which is again incompatible with experimental observations.

2. For steady-state shear flows, DE predicts the shear stress to be a non-monotonic function of the shear rate, which is a constitutive instability. It also predicts the first normal stress to approach a constant value as the shear rate increases. Experimental data seem to show an almost constant but slowly increasing shear stress and an ever-increasing first normal stress difference.

3. For small shear deformations, DE underestimates the value of the normal stress ratio $-N_{2} / N_{1}$ : its zero-strain limit is $1 / 7=0.142$, while experiments show values around 0.25 [5].

It has been shown, [4] that the first discrepancy can be removed at high shear rates by consideration of chain stretch effects. Several models including this feature have been proposed. Recently, Ianniruberto and Marrucci [6] suggested that a mechanism called convective constraint release (CCR) might explain the observed monotonic growth of the shear stress. This mechanism takes into account the convection of the entanglements along the polymer chain due to the flow. At high flow rate $\left(\tau_{\mathrm{d}} \dot{\gamma}>1\right.$, where $\tau_{\mathrm{d}}$ is the reptation time constant), the convective removal of entanglements induces a faster relaxation of the chain at increasing shear rates, thus explaining the observed growth of the shear stress. Marrucci et al. [7] also suggested that the discrepancy concerning the normal stress ratio might come from an inappropriate strain measure in the basic DE theory. They showed that a new strain measure, derived from force balance requirements at the nodes of a simple three-chain network, correctly predicts the value for the normal stress ratio in a step strain experiment. The CCR mechanism and the new strain measure have both been implemented recently by Marrucci et al. [1] in the so-called MGI constitutive equation. The latter has an integral form which is very well approximated by a differential constitutive equation. The behaviour of the MGI model in complex flows has been studied recently by Wapperom and Keunings [8].

Recent constitutive equations such as the MGI model introduce an additional coupling between the velocity gradient and the conformation variables, whose thermodynamic consistency needs to be verified. In the present work, using the single generator bracket formalism of non-equilibrium thermodynamics [9], we build a thermodynamically consistent constitutive equation of the differential type for linear entangled polymers. The starting point of our developments is the MGI differential model. We show that non-equilibrium thermodynamics suggests the introduction of an additional term in the relation between the stress and the conformation variables of the MGI model. The proposed constitutive equation has an additional parameter and contains the consistent MGI model as a particular case. Its behaviour in steady and transient shear flow is shown to be in qualitative agreement with experimental observations. 


\section{Non-equilibrium thermodynamics}

The compatibility of constitutive equations with non-equilibrium thermodynamics, as developed through the single generator bracket [9] or the two generator GENERIC [10] formalisms, ensures the satisfaction of symmetry constraints for the coupling parameters describing the dissipation in the linear limit (Onsager/Casimir reciprocal relation [11]). It also ensures a non-negative entropy production.

We consider differential constitutive equations for incompressible isothermal flows in the context of the single generator bracket formalism [9] of non-equilibrium thermodynamics. In order to describe the behaviour of a fluid with an internal microstructure, one has few but important choices to make:

- The variables of the problem. In addition to the velocity $v_{\alpha}$, we shall consider here a single second-order conformation tensor $c_{\alpha \beta}$ as an internal parameter describing the microstructure of the fluid. The physical meaning of the selected variables alone dictates the conservative convective component of the constitutive equation. In this case, the conservative part of the system follows Euler's equations of hydrodynamics with an additional component depending on $\boldsymbol{c}$, and an evolution equation given in the form:

$$
\stackrel{\nabla}{c}=0
$$

where $\left(\nabla^{\nabla}\right)$ is the upper-convected time derivative [12]. Therefore, in the absence of dissipation, $\boldsymbol{c}$ can be interpreted as the Finger strain tensor, the governing equations reducing to those for large deformation elasticity [13].

- The extended Helmholtz free energy density a of the system as a function of the selected variables and possibly their gradients. Here, we consider that the gradient of the conformation tensor does not influence the free energy. At equilibrium, $a$ reduces to the thermodynamic free energy, which is obtained when $a$ is minimised with respect to the internal parameters. Away from equilibrium, the gradient of $a$ is, in general, non-zero and acts like a thermodynamic driving force.

- The structure of dissipative phenomena, related for example to CCR and the relaxation of the microstructure.

For a viscoelastic constitutive model described by a single second-order internal tensor parameter, the addition of dissipative phenomena described by the lowest possible order (quadratic) interaction terms yields the following general constitutive equation [9]

$$
\begin{aligned}
& \frac{\partial c_{\alpha \beta}}{\partial t}=-v_{\gamma} \nabla_{\gamma} c_{\alpha \beta}+c_{\alpha \gamma} \nabla_{\gamma} v_{\beta}+c_{\gamma \beta} \nabla_{\gamma} v_{\alpha} \\
&-\Lambda_{\alpha \beta \gamma \epsilon} \frac{\partial a}{\partial c}+L_{\alpha \beta \epsilon \epsilon} \nabla_{\gamma} v_{\epsilon}, \\
& \sigma_{\alpha \beta}=2 c_{\beta \gamma} \frac{\partial a}{\partial c_{\alpha \gamma}}+L_{\alpha \beta \gamma \epsilon} \frac{\partial a}{\partial c_{\gamma \epsilon}}+Q_{\alpha \beta \gamma \epsilon} \nabla_{\gamma} v_{\epsilon} .
\end{aligned}
$$

Eq. (1) describes the time evolution of the conformation tensor $\left(c_{\alpha \beta}\right)$, while Eq. (2) expresses the stress as a function of the system's variables and of the derivative of the extended free energy with respect to these variables. On the first line of Eq. (1), we recognise the upper-convected time derivative of the conformation tensor, while on the second line we find two dissipative terms. The first accounts for relaxation phenomena, while the second is a mixed term leading, e.g. to the mixed-convected time 
derivative in the Johnson/Segalman fluid model [9]. The three terms on the right-hand side of Eq. (2) can be respectively interpreted as a conservative term, similar to what is obtained in non-linear elasticity, a mixed correction term, and a viscous dissipation term. The fourth-order tensors $\Lambda_{\alpha \beta \gamma \epsilon}, L_{\alpha \beta \gamma \epsilon}$ and $Q_{\alpha \beta \gamma \epsilon}$ arise in the limit of linear irreversible thermodynamics [14] and come from a first-order approximation of general dissipative phenomena. These tensors are phenomenological and can depend upon the variables of the problem but not their derivatives. They also need to be invariant to the following permutations of indices

$$
\alpha \beta \gamma \epsilon \leftrightarrow \beta \alpha \gamma \epsilon \leftrightarrow \alpha \beta \epsilon \gamma \leftrightarrow \beta \alpha \epsilon \gamma .
$$

Appropriate choices for the extended free energy and the dissipative structure yield well known constitutive equations such as the UCM, Johnson/Segalman or Giesekus models [9].

At this point, it should be noticed that Eqs. (1) and (2), as presented in [9], were obtained for a tensor $L_{\alpha \beta \gamma \epsilon}$ symmetric with respect to an exchange of the first two indices by the last two, $\alpha \beta \leftrightarrow \gamma \epsilon$. In Appendix A, we show that these equations remain valid for a general tensor $L_{\alpha \beta \gamma \epsilon}$ where this symmetry is not obeyed.

In the following sections, we shall first present the physical phenomena we wish to include in the constitutive equation, and then we shall consider the way to express these in a form compatible with the single generator bracket formalism.

\section{Convective constraint release and force balance}

It is not a surprise that constitutive equations that consider reptation as the only source of dissipation are unable to predict a monotonic growth of the steady-state shear stress as a function of shear rate. Indeed, at increasing flow rates, the thermal relaxation terms of these equations can be considered frozen, compared to the convective part. The relatively slow relaxation of the tube segments cannot counteract alignment in the shear direction. This explains why one observes a decrease of the shear stress at increasing shear rates greater than $1 / \tau_{\mathrm{d}}[6]$.

CCR takes into account the removal of entanglements due to their convection by the flow along the polymer chain. In this fashion, entanglements are destroyed once they are convected past the end of the chain. Considering that classical relaxation and flow-induced CCR operate in parallel, it is possible to sum their frequencies. With the assumption that the tube renewal frequency caused by CCR is proportional to the rate of convection along the tube, Ianniruberto and Marrucci [6] obtained a modified relaxation time $\tau$, defined as

$$
\frac{1}{\tau}=\frac{1}{\tau_{\mathrm{d}}}+\beta \boldsymbol{k}:\langle\boldsymbol{u} \boldsymbol{u}\rangle,
$$

where $\tau_{\mathrm{d}}$ is the DE disengagement time, $\langle\boldsymbol{u} \boldsymbol{u}\rangle$ the orientation tensor, $\boldsymbol{k}$ the transpose of the velocity gradient and $\beta$ denotes an adjustable scalar parameter. Although a value for $\beta$ of unity seems more natural, it has been argued [6] that $\beta$ should be somewhat greater than unity. One should also notice that $\boldsymbol{k}$ : $\langle\boldsymbol{u} \boldsymbol{u}\rangle$ is very similar to the rate of tube stretch found in models like those presented in [4]. Ianniruberto and Marrucci [6] showed that the CCR relaxation mechanism induces a monotonic growth of the shear stress and improves the agreement of the model with the Cox-Merz rule.

In order to correct the step strain predictions of DE, Marrucci et al. [7] suggested that a strain measure taking into account some requirement of force balance at the node of the entangled network should be 
adopted. Using a simple three-chain cubic network, they proposed a new strain measure that automatically fulfils the force balance at the entanglements of the network. For an elastic (non-relaxing) network, the new measure is

$$
\tilde{\boldsymbol{Q}}=\frac{\boldsymbol{C}^{1 / 2}}{\operatorname{Tr}\left(\boldsymbol{C}^{1 / 2}\right)},
$$

where $\boldsymbol{C}$ is the Finger tensor. For step strain deformations, just after the deformation, and before relaxation starts, the stress tensor is then given by

$$
\sigma=6 G_{N}^{(0)}\left(\tilde{Q}-\frac{1}{3} \delta\right)
$$

where $G_{N}^{(0)}$ is the plateau modulus and $\delta$ is the unit tensor.

\section{Constructing the model}

Relating the concepts of force balance and CCR to the bracket formalism is not straightforward and requires some insight. In this section, we present this process in a top-down approach.

Having selected a non-negative definite, symmetric second-order tensor $c$ as an additional internal variable, we see from Eqs. (1) and (2) that the remaining building blocks are as follows:

1. The specification of the extended Helmholtz free energy density $a$.

2. The development of the three fourth-order tensors $\boldsymbol{\Lambda}, \boldsymbol{L}$ and $\boldsymbol{Q}$.

Since the tensor $\boldsymbol{Q}$ accounts for viscous dissipation, its contribution will be neglected as we are interested in microstructure-induced stress. From Eq. (1), we find that the CCR mechanism described earlier can only be incorporated in the model through the mixed term $L_{\alpha \beta \gamma \epsilon} \nabla_{\gamma} v_{\epsilon}$. Indeed, in the lowest order expansion for the dissipation, only this term can provide a coupling between the velocity and the conformation tensor other than the upper-convected time derivative. Additional constraints between $\boldsymbol{\Lambda}$ and $\boldsymbol{L}$ will also appear from Eq. (4). In the absence of dissipative phenomena, the only degrees of freedom lie in the free energy density. This is where we incorporate the new strain measure (5).

\subsection{Conservative part}

In the absence of dissipative phenomena, the constitutive equation is uniquely determined by the form of the free energy density. From Eq. (1) we see that the evolution equation of the conformation tensor $c$ is compatible with its interpretation as the Finger strain tensor. In the absence of dissipation $(\boldsymbol{\Lambda}=\boldsymbol{L}=0)$, equating the remaining non-vanishing parts of the right-hand side of Eqs. (2) and (6) implies that the free energy density $a$ satisfies

$$
6 G_{N}^{(0)}\left(\frac{c^{1 / 2}}{\operatorname{Tr} c^{1 / 2}}-\frac{1}{3} \delta\right)=2 c \frac{\partial a}{\partial c},
$$

or

$$
\frac{\partial a}{\partial \boldsymbol{c}}=3 G_{N}^{(0)}\left(\frac{\boldsymbol{c}^{-1 / 2}}{\operatorname{Tr} c^{1 / 2}}-\frac{1}{3} c^{-1}\right) .
$$


This expression allows us to use the thermodynamic formalism in order to describe the same strain measure as proposed by Marrucci et al. Integrating Eq. (8), we find the following expression for the extended free energy density

$$
a(c)=6 G_{N}^{(0)}\left\{\ln \left(\operatorname{Tr} c^{1 / 2}\right)-\frac{1}{6} \ln (\operatorname{det} c)\right\} .
$$

A closer look at this expression shows that it is independent of the magnitude of $c$. Indeed, if we substitute $c$ for $\gamma c$ in Eq. (9), $\gamma$ being a positive scalar, we get

$$
\begin{aligned}
a(\gamma \boldsymbol{c}) & =6 G_{N}^{(0)}\left\{\ln \left(\gamma^{1 / 2} \operatorname{Tr} c^{1 / 2}\right)-\frac{1}{6} \ln \left(\gamma^{3} \operatorname{det} \boldsymbol{c}\right)\right\} \\
& =6 G_{N}^{(0)}\left\{\frac{1}{2} \ln \gamma+\ln \left(\operatorname{Tr} c^{1 / 2}\right)-\frac{1}{2} \ln \gamma-\frac{1}{6} \ln (\operatorname{det} c)\right\}=a(\boldsymbol{c}) .
\end{aligned}
$$

As $a$ is independent of the magnitude of $c$, and as $c$ is by definition equal to the unit tensor at equilibrium, $a$ should be minimised for $c=\gamma \delta$. For these values indeed, the gradient $\partial a / \partial c$ vanishes, while the second-order derivative of $a$ reduces to

$$
\frac{\partial^{2} a}{\partial c_{\mathrm{eq}}^{2}}=\frac{G_{N}^{(0)}}{3 \gamma^{4}} \delta \boldsymbol{\delta},
$$

which is a positive definite fourth-order tensor. Eq. (9) describes, therefore a thermodynamically valid free energy.

We can better understand the meaning of this free energy density if we rewrite it as

$$
a(\boldsymbol{c})=-G_{N}^{(0)} \ln \operatorname{det}\left(\frac{\boldsymbol{c}^{1 / 2}}{\operatorname{Tr} c^{1 / 2}}\right)^{2} .
$$

This shows that all stretching effects have been neglected through the scaling with the trace of $c^{1 / 2}$, and only an entropic orientational contribution remains (See Eq. 13C.7-7 p. 209 in [15], with $\boldsymbol{\alpha}=$ $3 \boldsymbol{c} / \operatorname{Tr} \boldsymbol{c})$. The modelling assumption is thus that the orientational distribution of the tube segments is described by $c^{1 / 2}$ rather than by $c$. As taking the square root of $c$ only changes the eigenvalues of the tensor, this can be interpreted as a reweighting of the eigenvectors of the conformation tensor.

\subsection{Dissipative part}

In the modelling of dissipative phenomena, we shall first focus on the relaxation tensor $\boldsymbol{\Lambda}$ of Eq. (1). Since the extended free energy density (9) is independent of the magnitude of $\boldsymbol{c}$, it is also most natural to keep this feature here and obtain an evolution equation for $c$ that would also be independent of its magnitude. Therefore, comparing Eqs. (1) and (8), we find that $\boldsymbol{\Lambda}$ should scale like $\boldsymbol{c}^{2}$. We shall then define two different relaxation tensors that satisfy the symmetry relations (3)

$$
\begin{aligned}
& \Lambda_{\alpha \beta \gamma \epsilon}^{(1)}=\frac{c_{\delta \delta}^{1 / 2}}{6 \tau_{\mathrm{d}} G_{N}^{(0)}}\left(c_{\alpha \gamma}^{1 / 2} c_{\beta \epsilon}+c_{\alpha \epsilon}^{1 / 2} c_{\beta \gamma}+c_{\beta \gamma}^{1 / 2} c_{\alpha \epsilon}+c_{\beta \epsilon}^{1 / 2} c_{\alpha \gamma}\right), \\
& \Lambda_{\alpha \beta \gamma \epsilon}^{(2)}=\frac{3}{6 \tau_{\mathrm{d}} G_{N}^{(0)}}\left(c_{\alpha \gamma} c_{\beta \epsilon}+c_{\alpha \epsilon} c_{\beta \gamma}+c_{\beta \gamma} c_{\alpha \epsilon}+c_{\beta \epsilon} c_{\alpha \gamma}\right) .
\end{aligned}
$$


The tensor $\boldsymbol{\Lambda}^{(1)}$ has been constructed to obtain a constitutive equation as close as possible to the MGI differential model proposed in [1] (see Appendix B). On the other hand, the tensor $\boldsymbol{\Lambda}^{(2)}$ can be seen in a naive way as the most natural tensor expression with a scaling like $\boldsymbol{c}^{2}$. This relaxation tensor is actually identical to the one found in the Giesekus model with the mobility factor equal to unity (see [9] p. 265 with $\alpha=1$ ). It is obtained from $\boldsymbol{\Lambda}^{(1)}$ using a mobility tensor equal to $3 \boldsymbol{c}^{1 / 2} / \operatorname{Tr} \boldsymbol{c}^{1 / 2}$ (see [9], pp. 252-256). The pre-factors in Eqs. (11) and (12) are such as to obtain the right linear viscoelastic limit and to keep the physical meaning of the parameters $\tau_{\mathrm{d}}$ and $G_{N}^{(0)}$ unchanged. Finally, we propose the relaxation tensor $\boldsymbol{\Lambda}$ to be a linear combination of $\boldsymbol{\Lambda}^{(1)}$ and $\boldsymbol{\Lambda}^{(2)}$

$$
\Lambda_{\alpha \beta \gamma \epsilon}=(1-\alpha) \Lambda_{\alpha \beta \gamma \epsilon}^{(1)}+\alpha \Lambda_{\alpha \beta \gamma \epsilon}^{(2)} .
$$

The phenomenological parameter $\alpha$ plays a role similar to that of the mobility factor in the Giesekus model [9].

\subsection{Introducing convective constraint release}

The dissipative term induced in Eq. (1) by the $\boldsymbol{\Lambda}$ tensor is independent of the velocity gradient $\boldsymbol{k}^{\mathrm{T}}$. The CCR mechanism (4), however, introduces an additional linear dependence on $\boldsymbol{k}$. The only way this can originate within the quadratic dissipation formalism is from a non-vanishing $\boldsymbol{L}$ tensor in Eq. (1). Moreover, by comparing Eqs. (1) and (4), we find that $\boldsymbol{L}$ must have the following form:

$$
L_{\alpha \beta \gamma \epsilon}=-J(\boldsymbol{c}, \boldsymbol{k}) \tau_{\mathrm{d}} \beta \Lambda_{\alpha \beta \delta \phi} \frac{\partial a}{\partial c} \delta \frac{c_{\gamma \epsilon}^{1 / 2}}{c_{\theta \theta}^{1 / 2}} \equiv J(\boldsymbol{c}, \boldsymbol{k}) L_{\alpha \beta \gamma \epsilon}^{*},
$$

where $J(\boldsymbol{c}, \boldsymbol{k})$ is an appropriate switch function which is equal either to 1 or 0 (see Appendix A).

At this time, we have to remark that, in contrast to the original quadratic dissipation theory (as described by Eqs. (1) and (2)), $\boldsymbol{L}$ is found here to be a function of the Volterra derivative of the Hamiltonian with respect to the internal parameter $\boldsymbol{c}$. Indeed, this is the only way through which the CCR mechanism can be introduced into the non-equilibrium formalism, i.e. by requiring a higher order non-linearity in the dissipation mechanism introduced by $\boldsymbol{L}$. What we therefore propose here is an "ansatz", sort of a mean field theory approach, with the form for $\boldsymbol{L}$ being suggested by rather than dictated from non-equilibrium thermodynamics (since the Onsager-Casimir relations from which the original term introduced by $\boldsymbol{L}$ in the dissipation originates are strictly applicable only for a quadratic dissipation close to the equilibrium limit). On the other hand, what non-equilibrium thermodynamics requires is that the overall rate of entropy production be non-negative. Since a non-symmetric $\boldsymbol{L}$ contributes to the entropy production (see Appendix A) and this contribution can be either positive or negative, and since it is not in general easy to a priori tailor the other contributions to guarantee in all cases an overall positive entropy production, we take the further step here to also propose a corrective multiplicative factor $J(\boldsymbol{c}, \boldsymbol{k})$ which acts as a "switch function" selectively turning the $\boldsymbol{L}$ term off when its individual contribution to the rate of entropy production is negative (see Appendix A for a full expression). This is certainly allowed, since $\boldsymbol{L}$ corresponds to a higher non-linearity anyhow. It only risks to be too conservative (eliminating CCR for conditions under which it may have been thermodynamically admissible), but we propose it anyhow for two reasons:

1. It is a thermodynamically-induced correction that it is relatively easy to implement and when implemented guarantees the thermodynamic consistency of the model, 
2. It is a correction that is applicable only rarely (most notably: during flow reversal) since in most flows, and certainly all the simple shear flows examined in the present work, this correction is not necessary as the corresponding rate for the entropy production term is positive (and thus $J(\boldsymbol{c}, \boldsymbol{k})=1$ ).

Finally, we note that a similar correction was found necessary to be introduced in the original MGI model [16] in connection to the physical interpretation of the CCR as a correction to the relaxation time for consistency, such a correction needs to be taken into account only when it is positive; if negative, it risks to make the overall relaxation time negative which is aphysical. However, note that here, and in contrast to the original MGI model, we have this on-off switch affecting both the stress and the evolution equation for $c$ in a concerted fashion.

A consequence of the introduction of a non-vanishing tensor $\boldsymbol{L}$ is that it brings an additional term in the stress Eq. (2). This term is of similar nature to the one that has to be introduced in the Johnson and Segalman model when a mixed time derivative is used instead of the upper-convected derivative. The presence of this term introduces significant changes in predictions for the extra-stress tensor.

\section{Completed model}

Combining all the building blocks presented above, we can derive a full model, which is thermodynamically-consistent and involves both the new strain measure and the CCR mechanism of the MGI model.

After substitution of Eq. (14) into Eqs. (1) and (2), the model reads

$$
\begin{aligned}
& \frac{\partial c_{\alpha \beta}}{\partial t}=-v_{\gamma} \nabla_{\gamma} c_{\alpha \beta}+c_{\alpha \gamma} \nabla_{\gamma} v_{\beta}+c_{\gamma \beta} \nabla_{\gamma} v_{\alpha} \\
&-\left(1+J(\boldsymbol{c}, \boldsymbol{k}) \tau_{\mathrm{d}} \beta \frac{c_{\delta \phi}^{1 / 2}}{c_{\theta \theta}^{1 / 2}} \nabla_{\delta} v_{\phi}\right) \Lambda_{\alpha \beta \gamma \epsilon} \frac{\partial a}{\partial c_{\gamma \epsilon}}, \\
& \sigma_{\alpha \beta}=2 c_{\beta \gamma} \frac{\partial a}{\partial c_{\alpha \gamma}}-J(\boldsymbol{c}, \boldsymbol{k}) \tau_{\mathrm{d}} \beta \Lambda_{\alpha \beta \delta \phi} \frac{\partial a}{\partial c_{\delta \phi}} \frac{c_{\gamma \epsilon}^{1 / 2}}{c_{\theta \theta}^{1 / 2}} \frac{\partial a}{\partial c_{\gamma \epsilon}},
\end{aligned}
$$

with $a, \boldsymbol{\Lambda}$ and $J(\boldsymbol{c}, \boldsymbol{k})$ defined respectively, by Eqs. (9), (13) and (A.3). On the second line of Eq. (15), we clearly see that all dissipative phenomena will occur with an apparent relaxation time corresponding to CCR, while the new strain measure appears in the first term of Eq. (16).

In the sequel, we focus on two limiting cases of the proposed model, obtained for $\alpha=0$ and 1 . For $\alpha=0$, the model reads

$$
\begin{aligned}
& \stackrel{\nabla}{c}=-2\left(\frac{1}{\tau_{\mathrm{d}}}+J(\boldsymbol{c}, \boldsymbol{k}) \beta \boldsymbol{k}: \frac{\boldsymbol{c}^{1 / 2}}{\operatorname{Tr} \boldsymbol{c}^{1 / 2}}\right) \operatorname{Tr} \boldsymbol{c}^{1 / 2}\left(\frac{\boldsymbol{c}}{\operatorname{Tr} \boldsymbol{c}^{1 / 2}}-\frac{1}{3} \boldsymbol{c}^{1 / 2}\right), \\
& \sigma=6 G_{N}^{(0)}\left(\frac{\boldsymbol{c}^{1 / 2}}{\operatorname{Tr} \boldsymbol{c}^{1 / 2}}-\frac{1}{3} \delta\right) \cdot\left(\boldsymbol{\delta}-J(\boldsymbol{c}, \boldsymbol{k}) \beta\left(3-\frac{1}{3} \operatorname{Tr} \boldsymbol{c}^{1 / 2} \operatorname{Tr} \boldsymbol{c}^{-1 / 2}\right) \frac{\boldsymbol{c}^{1 / 2}}{\operatorname{Tr} \boldsymbol{c}^{1 / 2}}\right) .
\end{aligned}
$$

It can be proved (see Appendix B) that this model is almost equivalent to the MGI differential model proposed by Marrucci et al. [1]. The evolution equation for the conformation tensor $c$ is identical. The MGI model, however, lacks the last factor in the expression of the extra stress tensor (18), which comes from the introduction of CCR into the model. Close to equilibrium, both models are very close as the 
missing term in the stress equation is only a high-order term. For $\alpha=0$, the proposed model is thus a thermodynamically-consistent version of the MGI model.

For $\alpha=1$, the model reads

$$
\begin{aligned}
& \stackrel{\nabla}{\boldsymbol{c}}=-6\left(\frac{1}{\tau_{\mathrm{d}}}+J(\boldsymbol{c}, \boldsymbol{k}) \beta \boldsymbol{k}: \frac{\boldsymbol{c}^{1 / 2}}{\operatorname{Tr} \boldsymbol{c}^{1 / 2}}\right)\left(\frac{\boldsymbol{c}^{3 / 2}}{\operatorname{Tr} \boldsymbol{c}^{1 / 2}}-\frac{1}{3} \boldsymbol{c}\right), \\
& \sigma=6 G_{N}^{(0)}\left(\frac{\boldsymbol{c}^{1 / 2}}{\operatorname{Tr} \boldsymbol{c}^{1 / 2}}-\frac{1}{3} \boldsymbol{\delta}\right) \cdot\left(\boldsymbol{\delta}-3 J(\boldsymbol{c}, \boldsymbol{k}) \beta\left(3-\frac{1}{3} \operatorname{Tr} \boldsymbol{c}^{1 / 2} \operatorname{Tr} \boldsymbol{c}^{-1 / 2}\right)\left(\frac{\boldsymbol{c}^{1 / 2}}{\operatorname{Tr} c^{1 / 2}}\right)^{2}\right) .
\end{aligned}
$$

This new model incorporates both CCR and the new strain measure as in the MGI model, but the structure of dissipative phenomena away from equilibrium, as shown in the next section, is quite different.

\section{Model predictions}

In this section, we compare the above models in various transient and steady-state shear flows. In all cases, the adjustable parameter $\beta$ has been set to unity as suggested in [6].

\subsection{Step strain in shear}

The normal stress ratio is an inherent feature of a model which, for step strain experiments, cannot be adjusted through a superposition of several modes; its value, therefore represents a good test. Fig. 1 shows the normal stress ratio $\left(-N_{2} / N_{1}\right)$ after a step strain in shear, as a function of the applied strain. These results were obtained numerically by applying a shear rate of large magnitude over a small time interval $\Delta t$, until a limit was reached for $\Delta t \rightarrow 0$. The experimental data are taken from Olson et al.

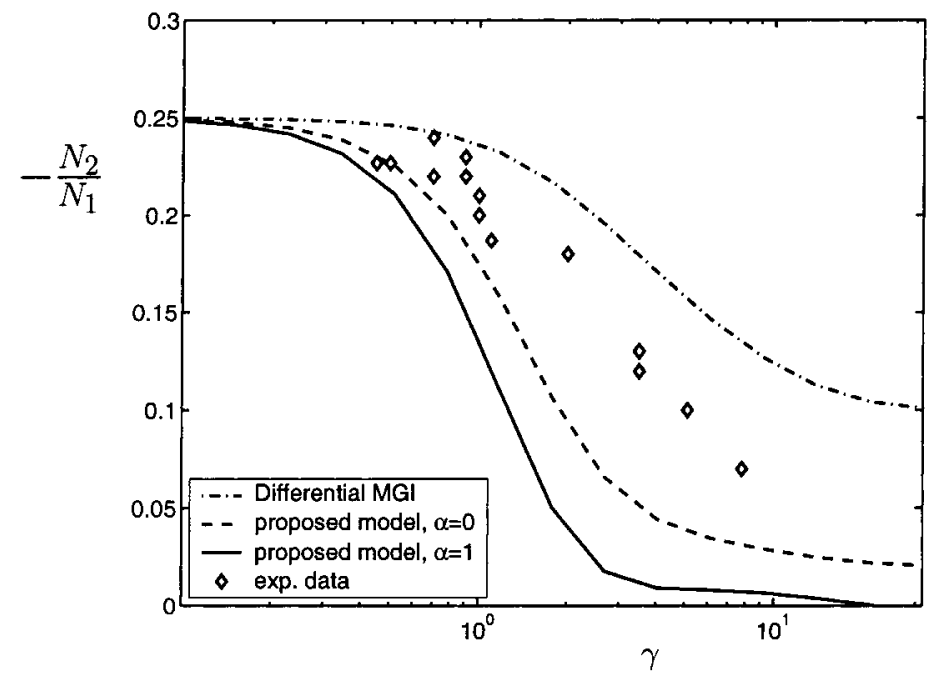

Fig. 1. Normal stress ration as a function of strain. Experimental data for a polystyrene solution and a poly-isoprene melt are reported from [5]. 


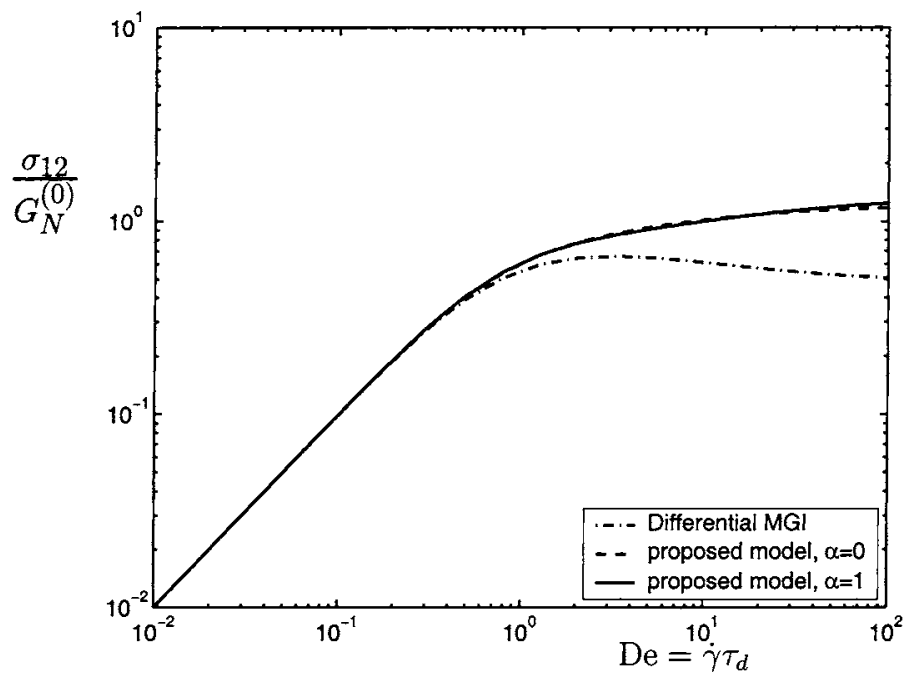

Fig. 2. Dimensionless shear stress as a function of the Deborah number.

[5]. As imposed by the strain measure (6) through the free energy (9), all three models predict a limit $-N_{2} / N_{1}=0.25$ for small strains. At higher strains, however, the MGI model does seem to reach a plateau at somewhat too large a value. The proposed model, on the other hand, exhibits too strong a decrease as the strain increases. A criterion for choosing between the models would be the value of the normal stress ratio at high strains, if such data were available experimentally.

\subsection{Steady-state shear flow}

As shown in Fig. 2, the new model displays a monotonic increase of the shear stress a function of the shear rate, with a unit value for $\beta$. On the other hand, it has been shown [1] that $\beta$ needs to be greater than 3.8 in order to obtain the same feature with the MGI model. Moreover, it should be noted that, at high shear rates, the proposed model displays shear stresses approaching a plateau value very close to $G_{N}^{(0)}$, which is in good agreement with the Cox-Merz rule.

The steady-state values of the first normal stress, shown in Fig. 3, show a major difference between the models. While the new model with $\alpha=0$ and MGI predict the first normal stress difference to reach a plateau, the new model with $\alpha=1$ predicts an ever-increasing curve. Such a behaviour is in agreement with experimental data [4]. Moreover, it should be recalled that no chain stretching effects have been introduced in any of these models.

In Fig. 4, we report the predictions of the various models as well as the experimental data for the normal stress ratio obtained by Kalogrianitis and van Egmond [17] in steady shear flow. Even though the MGI model predicts values which seem to fall in the right range, it does not predict the right slope, as the shear rate increases. The proposed model predictions for $\alpha=0$ and 1 however provide an envelope for the experimental data. Interestingly, the asymptotic limit of the normal stress ratio is a function of the $\alpha$-parameter. For $\alpha=1$, the limiting ratio is $1 / 2$ in shear flow, while it is 0.25 in step strain (Fig. 1). These numerical predictions have also been checked with an asymptotic analytical solution of the governing equations at small $D e$. 


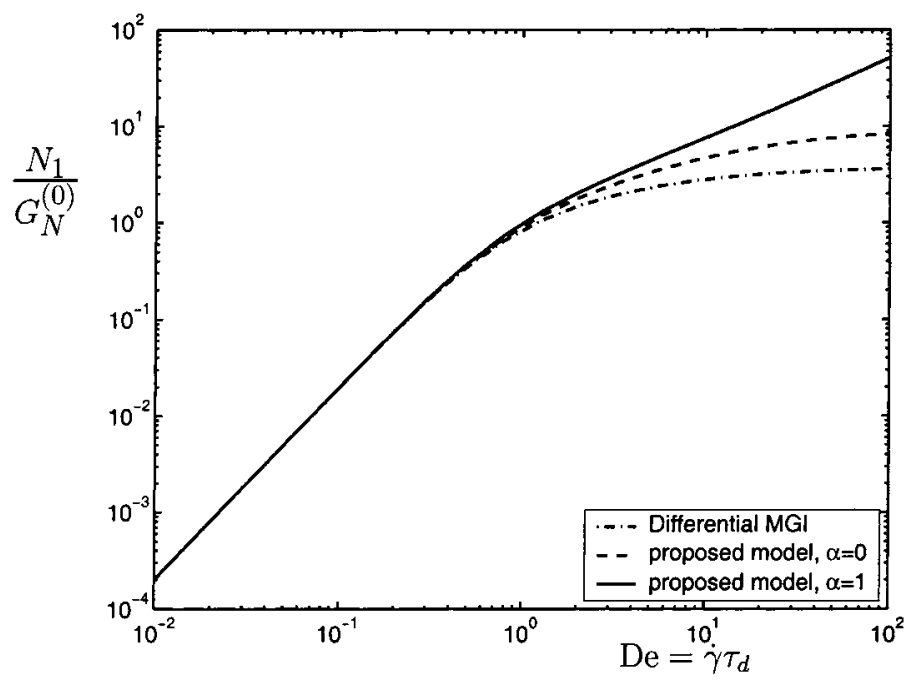

Fig. 3. Dimensionless first normal stress difference as a function of the Deborah number.

\subsection{Startup and cessation of shear flow}

In this section, we consider a non-trivial transient flow, namely the startup of shear flow followed by its sudden cessation. The simulation results should be compared qualitatively with the extensive set of experiments carried out by Kalogrianitis and van Egmond [17] on an entangled semi-dilute high molecular weight polystyrene solution. Among the features reported by these authors, we note an overshoot in the first normal stress difference upon inception of the flow, as well as an overshoot in the second normal

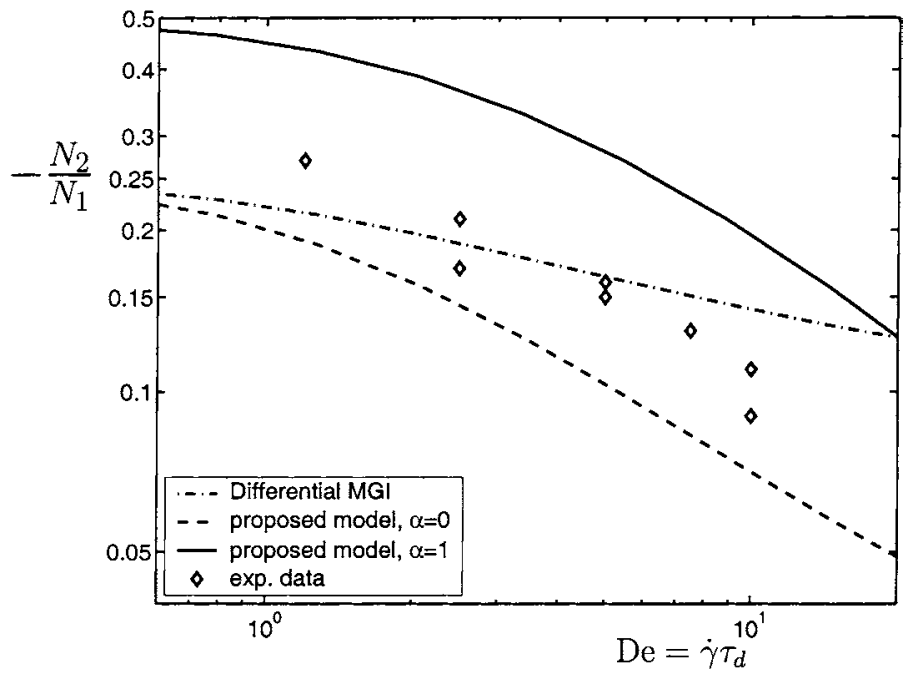

Fig. 4. Normal stress ratio as a function of the Deborah number. Experimental data for a polystyrene solution are reported from [17]. 


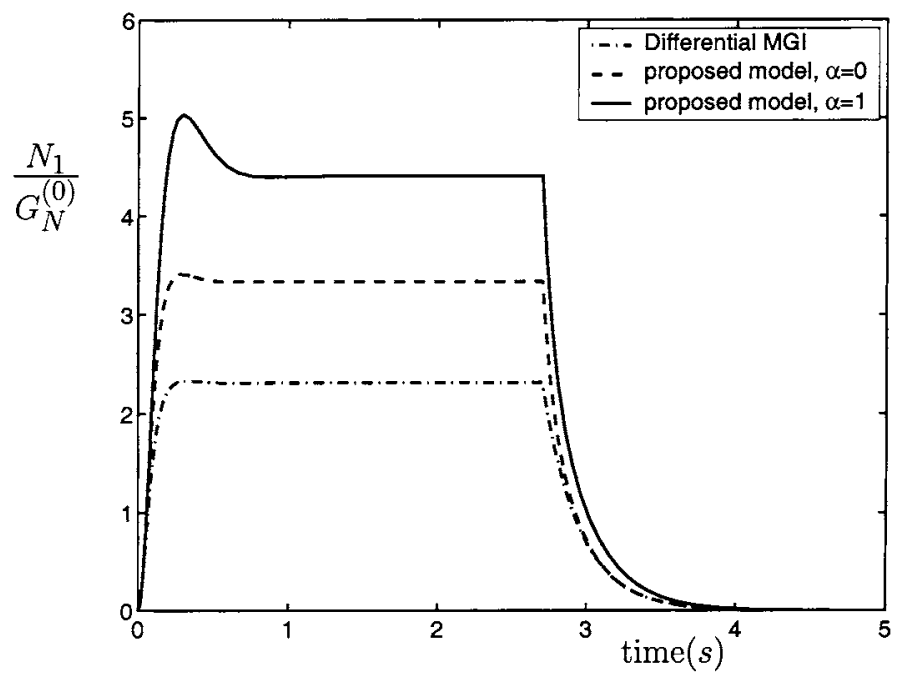

Fig. 5. Transient first normal stress difference as a function of time. As reported in [17], a strain rate of $20 \mathrm{~s}^{-1}$ is applied between time 0 and $2.7 \mathrm{~s}$, and $\tau_{\mathrm{d}}=0.25 \mathrm{~s}$.

stress difference both upon inception and cessation of the flow. Another important observation is that the relaxation of the normal stress ratio follows a single curve, independent of the shear rate previously applied. Also, the relaxation is non-exponential and the normal stress ratio approaches a constant value of about 0.9 at long times.

As shown in Fig. 5, all models display an overshoot in the first normal stress difference upon inception of the flow, but it is only significant for the new model with $\alpha=1$.

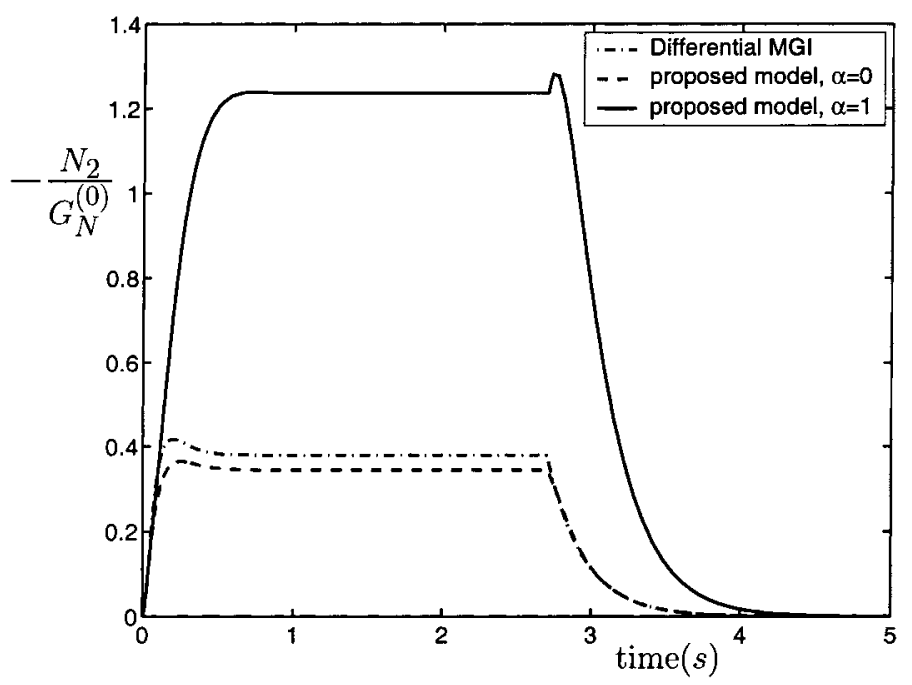

Fig. 6. Transient second normal stress difference as a function of time. As reported in [17], a strain rate of $20 \mathrm{~s}^{-1}$ is applied between time 0 and $2.7 \mathrm{~s}$, and $\tau_{\mathrm{d}}=0.25 \mathrm{~s}$. 


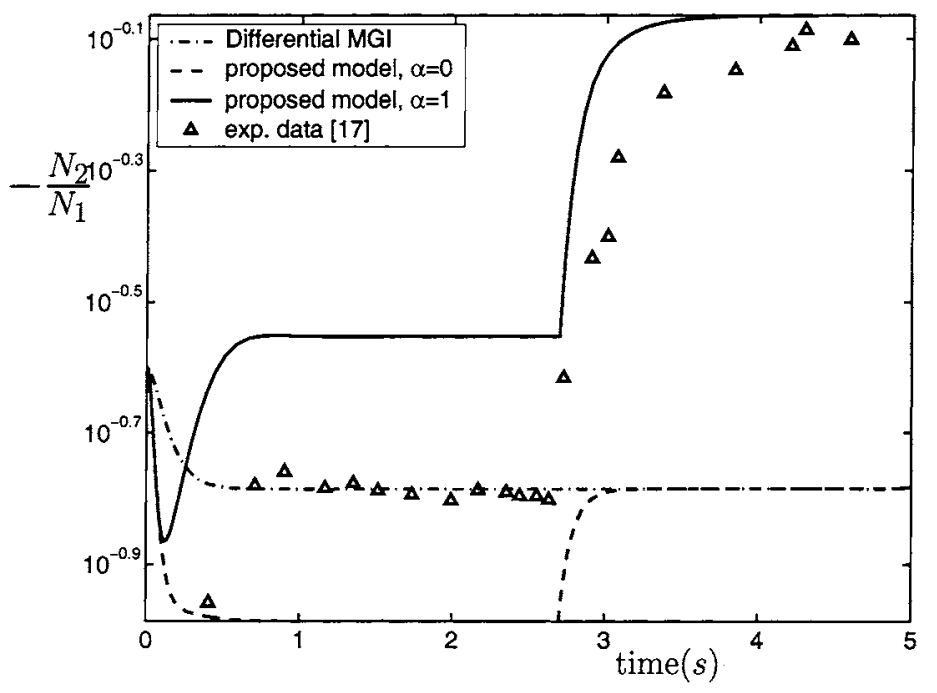

Fig. 7. Transient normal stress ratio as a function of time. As reported in [17], a strain rate of $20 \mathrm{~s}^{-1}$ is applied between time 0 and $2.7 \mathrm{~s}$, and $\tau_{\mathrm{d}}=0.25 \mathrm{~s}$.

Predictions of the second normal stress difference (Fig. 6) also show that the best behaviour is provided by the new model with $\alpha=1$. Indeed, only this model predicts an overshoot upon cessation of the shear flow, in agreement with experimental observations [17].

The evolution of the normal stress ratio is shown in Fig. 7. First, we see that all the models predict the same limit at small deformations but reach different steady-state values. Upon cessation of the flow,

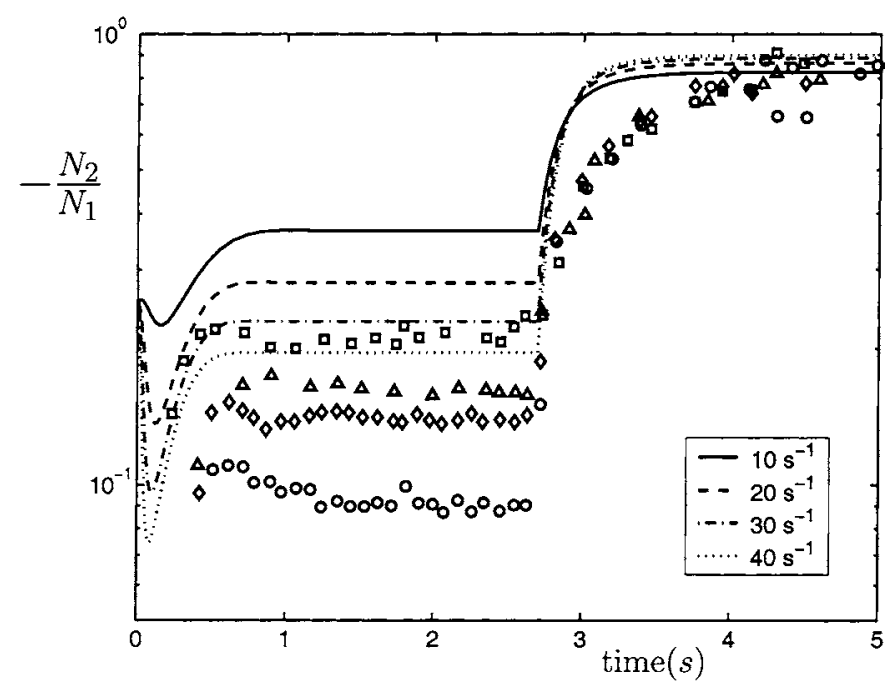

Fig. 8. Transient normal stress ratio as a function of time for the proposed model with $\alpha=1$. Corresponding experimental data taken from [17]. 
only the new model with $\alpha=1$ does predict the normal stress ratio to relax towards a value of 0.9 , as observed experimentally. Also, the normal stress ratio predicted by the MGI model is unaffected by the cessation of the flow, in contrast with its thermodynamically-consistent version (proposed model, $\alpha=0$ ). Of course these two models have the same response at large times.

In Fig. 8, we compare the transient predictions for the ratio $\left(-N_{2} / N_{1}\right)$ obtained with the proposed model against the experimental data of Kalogrianitis and van Egmond [17]. We see from there that the new model with $\alpha=1$ predicts the normal stress ratio to relax towards a high value of 0.9 , independently of the magnitude of the previously-applied shear rate. The very fast response upon inception of the flow is caused by the selected strain measure which enforces a value of 0.25 for small deformations.

\section{Conclusions}

Using the single generator bracket formalism of non-equilibrium thermodynamics, we propose here a new constitutive equation for linear entangled polymers that incorporates a simple version of the convective constraint release as well as a new strain measure proposed by Marrucci et al. [7]. This new model reproduces the conservative behaviour of the MGI differential model also proposed by Marrucci et al. [1] but has additional dissipative terms, in order to incorporate in a thermodynamically consistent fashion the convective constraint release mechanism. In steady shear flow, the new model predicts shear and normal stresses in good qualitative agreement with available experimental data. In transient shear flow, the proposed model is able to predict the qualitative behaviour of the normal stresses, especially during relaxation, after cessation of the flow.

Since these results were achieved using a single conformation tensor and with relatively simple expressions for relaxation and convective constraint release, the use of the single generator bracket formalism is considered quite encouraging for further refinements which could make the model more quantitatively correct.

\section{Acknowledgements}

This work is supported by the ARC 97/02-210 project, Communauté Française de Belgique. A.N. Beris would like to acknowledge a Fulbright Research Sabbatical Fellowship.

\section{Appendix A}

In this work, we considered an extended dissipative bracket, with respect to the one presented in [9]. For two arbitrary functionals $F$ and $G$, the new dissipative bracket based on general quadratic contributions in terms of the Volterra derivatives of $F$ and $G$ with respect to $c$ and $\boldsymbol{M}$ reads

$$
\begin{aligned}
{[F, G]=} & -\int_{\Omega} \Lambda_{\alpha \beta \gamma \epsilon} \frac{\delta F}{\delta c_{\alpha \beta}} \frac{\delta G}{\delta c_{\gamma \epsilon}} \mathrm{d}^{3} x-\int_{\Omega} Q_{\alpha \beta \gamma \epsilon} \nabla_{\alpha} \frac{\delta F}{\delta M_{\beta}} \nabla_{\gamma} \frac{\delta G}{\delta M_{\epsilon}} \mathrm{d}^{3} x \\
& -\int_{\Omega} L_{\alpha \beta \gamma \epsilon} \nabla_{\alpha} \frac{\delta F}{\delta M_{\beta}} \frac{\delta G}{\delta c_{\gamma \epsilon}}-L_{\gamma \epsilon \alpha \beta} \nabla_{\alpha} \frac{\delta G}{\delta M_{\beta}} \nabla_{\alpha} \frac{\delta G}{\delta M_{\beta}} \frac{\delta F}{\delta c_{\gamma \epsilon}} \mathrm{d}^{3} x+\text { entropy correction }
\end{aligned}
$$


where, $\delta \cdot / \delta \cdot$ is the Volterra derivative, $M$ the momentum and $\Omega$ the flow domain. In this new expression, only the last term changes from Eq. (8.1-5) in [9]. When the tensor $L_{\alpha \beta \gamma \epsilon}$, is symmetric upon exchange of $\alpha \beta$ with $\gamma \epsilon$, both expressions are identical. However, this new bracket allows the incorporation of a more general $c$-velocity coupling which, in the linear regime, leads to flux-potential relations that remain compatible with the extended Onsager/Casimir reciprocal relations. Direct identification leads to the dissipative terms of Eqs. (1) and (2).

It is interesting to note here that in the last term of (A.1), only the anti-symmetric part of $L_{\alpha \beta \gamma \epsilon}$ (w.r.t. $\alpha \beta \leftrightarrow \gamma \epsilon$ ) brings a contribution to the entropy production, while its corresponding term in the bracket (8.1-5) of [9] was not producing any entropy. The magnitude of the $\boldsymbol{L}$ term needs therefore to be tailored so that it always leads to a non-negative entropy production. This is achieved by making it proportional to a switch function $J(\boldsymbol{c}, \boldsymbol{k})$, which turns it off as soon as its rate of entropy production becomes negative. From [9], we know that the entropy production is given by

$$
\frac{\partial s}{\partial t}=-\frac{1}{T}[H, H]_{\mathrm{wec}},
$$

where $s$ is the entropy functional, $T$ the temperature, $H$ the Hamiltonian of the system, and the subscript wec means "without entropy correction". This immediately yields the following expression for $J(\boldsymbol{c}, \boldsymbol{k})$ :

$$
J(\boldsymbol{c}, \boldsymbol{k})=\frac{1}{2}\left(1+\frac{L_{\alpha \beta \gamma \epsilon}^{*}\left(\nabla_{\alpha} v_{\beta}\left(\partial a / \partial c_{\gamma \epsilon}\right)-\nabla_{\gamma} v_{\epsilon}\left(\partial a / \partial c_{\alpha \beta}\right)\right)}{\left|L_{\alpha \beta \gamma \epsilon}^{*}\left(\nabla_{\alpha} v_{\beta}\left(\partial a / \partial c_{\gamma \epsilon}\right)-\nabla_{\gamma} v_{\epsilon}\left(\partial a / c_{\alpha \beta}\right)\right)\right|}\right),
$$

which is either equal to 1 or 0 .

We believe, however that further developments of the convective constraint release theory might lead to a formulation of a new dissipation bracket where phenomenological corrections such as this would not be necessary anymore.

\section{Appendix B}

The differential MGI model proposed by Marrucci et al. [1] has the following form:

$$
\begin{aligned}
& \boldsymbol{T} \cdot \frac{\mathrm{D} \boldsymbol{T}}{\mathrm{D} t}+\frac{\mathrm{D} \boldsymbol{T}}{\mathrm{D} t} \cdot \boldsymbol{T}=\boldsymbol{k} \cdot \boldsymbol{T}^{2}+\boldsymbol{T}^{2} \cdot \boldsymbol{k}^{\mathrm{T}}-2 \boldsymbol{T}^{2}\left(\boldsymbol{k}: \frac{\boldsymbol{T}}{G}\right)-2 \frac{\boldsymbol{T}}{\tau} \cdot\left(\boldsymbol{T}-\frac{G}{3} \boldsymbol{\delta}\right), \\
& \boldsymbol{\sigma}=\boldsymbol{T}-\frac{G}{3} \boldsymbol{\delta}, \\
& \frac{1}{\tau}=\frac{1}{\tau_{\mathrm{d}}}+\beta \boldsymbol{k}: \frac{\boldsymbol{T}}{G},
\end{aligned}
$$

where $\boldsymbol{k}$ is the transpose of the velocity gradient, $(\mathrm{D} \cdot / \mathrm{D} t)$ is the material derivative and $G=6 G_{N}^{(0)}$. In the absence of dissipative phenomena, $\boldsymbol{T}$ is linked to the Finger strain tensor $\boldsymbol{C}$ by the following relation:

$$
\boldsymbol{T}=G \frac{\boldsymbol{C}^{1 / 2}}{\operatorname{Tr} \boldsymbol{C}^{1 / 2}}
$$


In order to relate this constitutive equation to the proposed model with $\alpha=0$, we shall start from the evolution Eq. (1) of the conformation tensor $c$

$$
\frac{\mathrm{D} \boldsymbol{c}}{\mathrm{D} t}=\boldsymbol{k} \cdot \boldsymbol{c}+\boldsymbol{c} \cdot \boldsymbol{k}^{\mathrm{T}}+\boldsymbol{\Omega},
$$

where $\boldsymbol{\Omega}$ represents the dissipative terms. When $\boldsymbol{\Omega}$ can be neglected, $\boldsymbol{c}$ becomes the Finger strain tensor. Defining $\boldsymbol{q}$ as

$$
\boldsymbol{q}=\boldsymbol{c}^{1 / 2}
$$

we find the equation describing the evolution of the trace of $\boldsymbol{q}$ by taking the contraction of Eq. (A.8) with $q^{-1}$

$$
\frac{\mathrm{DTr} \boldsymbol{q}}{\mathrm{D} t}=\boldsymbol{k}: \boldsymbol{q}+\frac{1}{2} \boldsymbol{q}^{-1}: \boldsymbol{\Omega} .
$$

Writing $\boldsymbol{T}$ as $\boldsymbol{T}=G(\boldsymbol{q} / \operatorname{Tr} \boldsymbol{q})$, we have

$$
\frac{\mathrm{D} \boldsymbol{T}}{\mathrm{D} t}=\frac{G}{\operatorname{Tr} \boldsymbol{q}} \frac{\mathrm{D} \boldsymbol{q}}{\mathrm{D} t}-\frac{G}{(\operatorname{Tr} \boldsymbol{q})^{2}} \frac{\mathrm{D} \operatorname{Tr} \boldsymbol{q}}{\mathrm{D} t} \boldsymbol{q} .
$$

Making use of Eqs. (A.10) and (A.11), we obtain an evolution equation for $\boldsymbol{T}$

$$
\boldsymbol{T} \cdot \frac{\mathrm{D} \boldsymbol{T}}{\mathrm{D} t}+\frac{\mathrm{D} \boldsymbol{T}}{\mathrm{D} t} \cdot \boldsymbol{T}=\boldsymbol{k} \cdot \boldsymbol{T}^{2}+\boldsymbol{T}^{2} \cdot \boldsymbol{k}^{\mathrm{T}}-2 \boldsymbol{T}^{2}\left(\boldsymbol{k}: \frac{\boldsymbol{T}}{G}\right)-\frac{\boldsymbol{q}^{-1}: \boldsymbol{\Omega}}{\operatorname{Tr} \boldsymbol{q}} \boldsymbol{T}^{2}-\left(\frac{G}{\operatorname{Tr} \boldsymbol{q}}\right)^{2} \boldsymbol{\Omega} .
$$

Identifying terms between Eqs. (A.4) and (A.12), we obtain the following expression for $\boldsymbol{\Omega}$

$$
\boldsymbol{\Omega}=-2\left(\frac{\operatorname{Tr} \boldsymbol{q}}{\tau_{\mathrm{d}}}+\beta \boldsymbol{k}: \boldsymbol{q}\right)\left(\frac{\boldsymbol{q}^{2}}{\operatorname{Tr} \boldsymbol{q}}-\frac{1}{3} \boldsymbol{q}\right) .
$$

The two dissipative contributions of Eq. (A.12) indeed reduce to

$$
\frac{\boldsymbol{q}^{-1}: \boldsymbol{\Omega}}{\operatorname{Tr} \boldsymbol{q}} \boldsymbol{T}^{2}=0, \quad\left(\frac{G}{\operatorname{Tr} \boldsymbol{q}}\right)^{2} \boldsymbol{\Omega}=-2 \frac{\boldsymbol{T}}{\tau} \cdot\left(\boldsymbol{T}-\frac{G}{3} \boldsymbol{\delta}\right) .
$$

The exact form of $\boldsymbol{\Lambda}^{(1)}$ is then obtained by comparing Eqs. (1), (8), (14) and (A.13). The expression Eq. (18) for the stress equation of the proposed model with $\alpha=0$ differs however from Eq. (A.5) as we are using a non-zero $\boldsymbol{L}$ tensor in the modelling of dissipation. Combining Eq. (2) with Eqs. (8),(11) and (14) we obtain the following stress equation for the new model $(\alpha=0)$

$$
\boldsymbol{\sigma}=\left(\boldsymbol{T}-\frac{G}{3} \delta\right) \cdot\left(\delta-\beta \frac{\boldsymbol{T}}{G}\left(3-\frac{G}{3} \operatorname{Tr} \boldsymbol{T}^{-1}\right)\right) .
$$

This expression clearly shows that the new term in the stress equation (compared with Eq. (A.5)) is a higher order term (order $\boldsymbol{T}^{2}$ ), which vanishes close to equilibrium. It should also be noticed that

$$
3-\frac{G}{3} \operatorname{Tr} \boldsymbol{T}^{-1}=0,
$$

at equilibrium. 


\section{References}

[1] G. Marrucci, F. Greco, G. Ianniruberto, Integral and differential constitutive equations for entangled polymers with simple versions of CCR and force balance on entanglements, Rheol. Acta 40 (2) (2001) 98-103.

[2] P.G. De Gennes, Reptation of a polymer chain in the presence of fixed obstacles, J. Chem. Phys. 55 (1971) $572-579$.

[3] M. Doi, S.F. Edwards, The Theory of Polymer Dynamics, Oxford University Press, 1986.

[4] D.W. Mead, R.G. Larson, M. Doi, A molecular theory for fast flows of entangled polymers, Macromolecules 31 (22) (1998) 7895-7914.

[5] D.J. Olson, E.F. Brown, W.R. Burghardt, Second normal stress difference relaxation in a linear polymer melt following step-strain, J. Polym. Sc. Part B 36 (14) (1998) 2671-2675.

[6] G. Ianniruberto, G. Marrucci, On compatibility of the Cox-Merz rule with the model of Doi and Edwards, J. Non-Newtonian Fluid Mech. 65 (2/3) (1996) 241-246.

[7] G. Marrucci, F. Greco, G. Ianniruberto, Simple strain measure for entangled polymers, J. Rheol. 44 (4) (2000) $845-854$.

[8] P. Wapperom, R. Keunings, Simulation of linear polymer melts in transient complex flow, J. Non-Newtonian Fluid Mech. 95 (1) (2000) 67-83.

[9] A.N. Beris, B.J. Edwards, Thermodynamics of Flowing Systems with Internal Microstructure. Oxford University Press, 1994.

[10] H.C. Öttinger, M. Grmela, Dynamics and thermodynamics of complex fluids. Part II. Illustration of a general formalism, Phys. Rev. E 56 (6) (1997) 6633-6655.

[11] S.R. De Groot, P. Mazur, Non-Equilibrium Thermodynamics. North Holland Publishing Company, Amsterdam, 1969.

[12] M. Grmela, Hamiltonian dynamics of incompressible elastic fluids, Phys. Lett. A 130 (1988) 81-86.

[13] B.J. Edwards, A.N. Beris, Noncanonical Poisson bracket for nonlinear elasticity with extensions to viscoelasticity, J. Phys. A: Math. Gen. 24 (1991) 2461-2480.

[14] G. Nicolis, Irreversible thermodynamics, Rep. Prog. Phys. 42 (1979) 225-268.

[15] R.B. Bird, C.F. Curtiss, R.C. Armstrong, O. Hassager, Dynamics of Polymeric Liquids, 2nd Edition, Vol. 2, Kinetic Theory. Wiley, New York, 1987.

[16] G. Marrucci, personal communication.

[17] S.G. Kalogrianitis, J.W. van Egmond, Full tensor optical rheometry of polymer fluids, J. Rheol. 41 (2) (1997) $343-364$. 